\title{
DYNAMICAL FRICTION EFFECTS ON THE MOTION OF STARS IN ROTATING SPHERICAL CLUSTERS
}

\author{
A. S. BARANOV and Yu. V.BATRAKOV \\ Institute of Theoretical Astronomy, Leningrad, U.S.S.R.
}

\begin{abstract}
Effects of dynamical friction on star orbits in a spherical cluster uniformly rotating with small angular velocity about a fixed axis are considered, deformations of the cluster due to the rotation being neglected. The test star is supposed to move in a noncircular restricted orbit under the influence of both the attraction of the cluster with the smoothed-out distribution of stellar matter and dynamical friction due to random encounters of the test star with other stars of the cluster.

The approximate formula for dynamical friction has been deduced, the encounters being supposed to be the binary ones. The differential equations for the osculating elements of the star orbit have been obtained for the two cases of the density distribution - the uniform and the exponential ones. The numerical results demonstrate the complicated character of dynamical friction effects on the evolution of the orbit. The orbit tends to become circular, and its inclination decreases. These effects are proportional to the mass of the test star. This leads to the conclusion that dynamical friction contributes roticeably to the concentration of massive stars near the center of the cluster.
\end{abstract}

\section{The Formulation of the Problem}

The field of force in the star cluster may be described as a superposition of the regular field defined by the continuous distribution of stellar matter in the cluster and of the irregular field defined by chance stellar encounters. When considering the star orbits the greatest attention is usually paid to the regular component of the force field, and the effect of stellar encounters as a rule, is entirely neglected. On this scheme each star follows the determinate trajectory under the sole influence of the smoothed-out gravitational field of the potential of the system as a whole, which is a function of the space coordinates only, the total energy of the star being unchanged, and the motion of the star being uniquely defined by its orbital parameters.

It is clear, however, that this determinate process of the motion of the star along a specified trajectory in the regular field will be disturbed when the test star passes close to other stars. Due to the accidental encounters with stars of the cluster an additional force arises, the magnitude, the direction and the duration of which depend on the initial conditions characterizing each single encounter. The knowledge of the position of the star and of the disturbing force acting on the latter at some instant of time gives only a certain probability of each possible position at the following instant of time. Therefore, the changes of the orbit under the influence of the encounters are random.

Relatively distant encounters of the test star with other stars of the cluster occur much more often than the close ones, and although the effect of a single distant encounter is quite small, the accumulated effect of distant encounters may result in an appreciable change of the star orbit. The negligibly small probability of the close encounters also enables us to disregard discontinuities in the behaviour of the stellar 
velocity as a random function of time and to consider the changes of the stellar velocity in the irregular field as a continuous random process.

As a result of the action of the irregular forces in gravitating systems, in particular, dynamical friction appears in the direction against the relative velocity of the test star. Dynamical friction in gravitating systems was for the first time investigated in detail by Chandrasekhar (1943b), but in his research the test star was assumed to move in a straight line in the structureless infinite medium. In real stellar systems the star orbits cannot, however, be considered rectilinear and must be restricted in space.

In this paper we deal with the influence of dynamical friction on the motion of the test star in a spherical cluster uniformly rotating with a small angular velocity around a fixed axis passing through the center of masses of the cluster. The deformation of the cluster caused by its rotation is considered to be neglected, when its influence on the test star motion is concerned. Furthermore, we shall assume that there are no external forces acting on the motion of the test star.

\section{Dynamical Friction in Stellar Clusters}

If one neglects the probability of the close encounters it is necessary to consider the action of the irregular forces in the stellar cluster as a random continuous Markoff process (for the fixed state at present, the state of the system in future does not depend on its state in the past). In this supposition the force of dynamical friction can be expressed as

$$
\mathbf{F}=m\langle\Delta \mathbf{v}\rangle / \Delta t
$$

where $m$ is the mass of the test star, $\langle\Delta \mathbf{v}\rangle / \Delta t$ is the average increment of the velocity of this star during the time interval $\Delta t$, which is sufficiently long for many encounters to occur during this time, but sufficiently short for the velocity of the star not to change appreciably (Chandrasekhar, 1943a, b). We shall suppose, as is usually the case (Chandrasekhar, 1942), that each encounter may be idealized as an independent twobody problem. For the sake of simplicity we shall suppose that all the stars in the cluster have the same mass, $\bar{m}$, and the mass $m$ of the test star, which differs, in general, from the mass $\bar{m}$, is small as compared with the total mass of the cluster $N \bar{m}(N$ is the number of stars in the cluster). According to these assumptions the increment of the velocity per unit time may be represented in the form (Rosenbluth et al., 1957)

$$
\begin{aligned}
& \langle\Delta \mathbf{v}\rangle / \Delta t=\Gamma \operatorname{grad} h, \\
& \Gamma=4 \pi G^{2} \bar{m}^{2} \ln \left[\frac{L \sigma^{2}}{G(m+\bar{m})}\right], \\
& h(\mathbf{v})=\frac{m+\bar{m}}{\bar{m}} \int \frac{\psi\left(\mathbf{v}^{\prime}\right)}{\left|\mathbf{v}-\mathbf{v}^{\prime}\right|} \mathrm{d} \mathbf{v}^{\prime}
\end{aligned}
$$

where $G$ is the constant of gravitation, $L$ is the maximal encounter parameter in the two-body problem, which may be considered as the average distance between the 
stars (Chandrasekhar, 1942), $\sigma^{2}$ is the mean square velocity of the stars in the cluster, $\psi\left(\mathbf{v}^{\prime}\right)$ is the distribution function of the velocities, $\mathrm{d} \mathbf{v}^{\prime}$ is a volume element in the velocity space. The dependence of the logarithm on the velocity in (3) is weak, therefore, the quantity $\Gamma$ may be assumed to be constant. The denominator in (4) represents the velocity of the test star relative to an arbitrary cluster star. Equations (2)-(4) are valid in any coordinate system, but for the sake of convenience when calculating the function $h(v)$ the system of coordinates referred to the centroid of the given point is used.

We shall now suppose that the distribution function of the velocities is Maxwellian:

$$
\psi\left(v^{\prime}\right)=D\left(\frac{3}{2 \pi \sigma^{2}}\right)^{3 / 2} \exp \left(-\frac{3 v^{\prime 2}}{2 \sigma^{2}}\right)
$$

where $D$ denotes the number of stars per unit volume. Substituting (5) in (4) and using integral representation,

$$
\frac{1}{\left|\mathbf{v}-\mathbf{v}^{\prime}\right|}=\frac{1}{\sqrt{ } \pi} \int_{-\infty}^{+\infty}\left[\exp \left(-\left|\mathbf{v}-\mathbf{v}^{\prime}\right|^{2}\right) \xi^{2}\right] d \xi
$$

( $\xi$ is an auxiliary variable of integration) after some minor rearranging, we find

$$
\operatorname{grad} h=\frac{m+\bar{m}}{\bar{m}} \frac{D}{v^{3}}\left[\phi(s)-\phi^{\prime}(s)\right] \mathbf{v}
$$

Here $s$ is a variable defined by

$$
s^{2}=3 v^{2} / 2 \sigma^{2},
$$

and $\phi(s)$ and $\phi^{\prime}(s)$ are respectively the error function and its derivative;

$$
\phi(s)=\frac{2}{\sqrt{ } \pi} \int_{0}^{s} \exp \left(-\xi^{2}\right) \mathrm{d} \xi, \quad \phi^{\prime}(s)=\frac{2}{\sqrt{ } \pi} \exp \left(-s^{2}\right) .
$$

Using (2), (3) and (6), we obtain from (1)

$$
\mathbf{F}=-4 \pi G^{2} m \bar{m}(m+\bar{m})\left\{\ln \left[\frac{L \sigma^{2}}{G(m+\bar{m})}\right]\right\} \frac{D}{v^{3}}\left[\phi(s)-s \phi^{\prime}(s)\right] \mathbf{v} .
$$

The function $v(s)=\phi(s)-s \phi^{\prime}(s)$ increases monotonically from zero to unit as $s$ changes from zero to infinity. For the sake of simplicity in what follows we shall assume that the velocity of the test star is not too different from $\sigma$. We are interested in the approximate estimate of dynamical friction effects for comparatively small time intervals at which changes of function $v(s)$ are quite small. Expanding $v(s)$ in powers of the deviations of the velocity of the test star from the root mean square 
velocity of the cluster stars and taking into account only the first term of the expansion, we obtain

$$
\mathbf{F}=-F_{0} \frac{D}{v^{3}} \mathbf{v}
$$

where $F_{0}$ is a constant, which has the form

$$
F_{0}=4 \pi G^{2} m \bar{m}(m+\bar{m})\left\{\ln \left[\frac{L \sigma^{2}}{G(m+\bar{m})}\right]\right\}\left[\phi\left(\sqrt{ } \frac{3}{2}\right)-\sqrt{ } \frac{3}{2} \phi^{\prime}\left(\sqrt{ } \frac{3}{2}\right)\right] .
$$

It must be emphasized here that the right-hand side of (7) does not take into consideration unavoidable fluctuations of the force. The greater the mass of the test star and its velocity, the more correct it is to restrict ourselves to the determinate part of $\mathbf{F}$ given by (7). The estimates below do not claim to be quite accurate but they do reveal the main tendency in the evolution of the star orbits, the kinetic energy of which exceeds the average kinetic energy of the stars in the cluster.

\section{Dynamical Friction Effects on the Motion of the Star Inside a Rotating Homogeneous Spherical Cluster}

Let us consider a spherical cluster with constant density $D=D_{0}$ and suppose that in a certain appropriately chosen fixed frame of reference $(x, y, z)$ with the origin in the center of the sphere, the cluster rotates with a uniform angular velocity $\omega$ about the $z$-axis. The potential $U$ for the case under consideration takes the form

$$
U=-\frac{1}{2} \alpha r^{2},
$$

where $r$ is the distance of the test star from the center of the cluster, and

$$
\alpha=\frac{4}{3} \pi G \bar{m} D_{0} .
$$

It is well-known that the trajectory of the star in the force field (8) is an ellipse, the center of which coincides with the center of the sphere, and the period of the revolution of the star $\tau$ around the center of the sphere in the motion along this ellipse depends neither on the dimensions nor on the compression of the latter and is equal to $2 \pi / \alpha^{1 / 2}$. The equation of the trajectory takes the form

$$
r^{2}=\frac{q^{2}}{1+\kappa \cos 2 \varphi}
$$

where

$$
q=r_{p}(1+\kappa)^{1 / 2}, \quad \kappa=\frac{r_{a}^{2}-r_{p}^{2}}{r_{a}^{2}+r_{p}^{2}}
$$

where $r_{p}$ and $r_{a}$ are respectively the pericentric and apocentric distances of the test star, $\varphi$ is the angle between the radius vector and the direction to the pericenter. The 
quantity $\kappa$ characterizes the compression of the ellipse and in many respects is analogous to the eccentricity.

The undisturbed star orbit in the gravitational field (8) is completely defined by the two isolating integrals of the motion: the energy integral (scalar) and the momentum integral (vector), which respectively take the form, in terms of peri- and apocentric distances,

$$
\begin{aligned}
\dot{r}^{2}+\alpha r^{2} & =\alpha\left(r_{p}^{2}+r_{a}^{2}\right), \\
\mathbf{r} \times \dot{\mathbf{r}} & =\mathbf{c},
\end{aligned}
$$

where dots denote the derivatives with respect to time. The following designations are used in (12):

$$
\begin{aligned}
& \mathbf{c}=c\left\{\begin{array}{c}
\sin i \sin \Omega \\
-\sin i \cos \Omega \\
\cos i
\end{array}\right\}, \quad c=\alpha^{1 / 2} r_{p} r_{a} \\
& \mathbf{r}=r\left\{\begin{array}{l}
\cos u \cos \Omega-\sin u \sin \Omega \cos i \\
\cos u \sin \Omega+\sin u \cos \Omega \cos i \\
\sin u \sin i
\end{array}\right\} .
\end{aligned}
$$

Here, as usual, $i$ is the inclination of the orbit, $\Omega$ is the longitude of the ascending node, $u=\varphi+\varpi$, and $\boldsymbol{\varpi}$ is the distance of the pericenter from the node.

For investigating the disturbed motion of the star in a rotating cluster it is necessary to keep in mind that $\mathbf{v}$ in (7) is the relative velocity, and it is necessary to calculate it according to the formula

$$
\mathbf{v}=\dot{\mathbf{r}}-\mathbf{w},
$$

where $\mathbf{w}$ is the linear velocity of the rotation of the cluster at the test star position.

To take into account the effects of the disturbing force (7) on the motion defined by (8), (10) and (12) we shall now consider $r_{p}, r_{a}, i, \Omega, \varpi$ to be the osculating variables. It means that the space coordinates and the components of the velocity in the disturbed motion at each instant of time are calculated according to the formulae of the undisturbed motion. We shall compose the differential equations for these variables.

Using the momentum and the energy integrals of the undisturbed motion (12) and the equation of the disturbed motion

$$
\ddot{\mathbf{r}}=\operatorname{grad} U+\mathbf{F} / m,
$$

we find the following equations for $r_{p}$ and $r_{a}$ :

$$
\begin{aligned}
& \dot{r}_{p}=\frac{1}{r_{a}^{2}-r_{p}^{2}}\left[\frac{r_{a}}{\sqrt{\alpha m}}(\mathbf{r} \times \mathbf{F}) \frac{\mathbf{c}}{c}-\frac{r_{p}}{\alpha m}(\dot{\mathbf{r}} \cdot \mathbf{F})\right], \\
& \dot{r}_{a}=\frac{1}{r_{a}^{2}-r_{p}^{2}}\left[\frac{r_{a}}{\alpha m}(\dot{\mathbf{r}} \cdot \mathbf{F})-\frac{r_{p}}{\sqrt{\alpha} m}(\mathbf{r} \times \mathbf{F}) \frac{\mathbf{c}}{c}\right],
\end{aligned}
$$


where

$$
\begin{gathered}
(\mathbf{r} \times \mathbf{F})=-\frac{F_{0} D_{0}}{v^{3}}\left\{\mathbf{c}-\omega\left[-\mathbf{i} x z-\mathbf{j} y z+\mathbf{k}\left(x^{2}+y^{2}\right)\right]\right\} \\
(\dot{\mathbf{r}} \cdot \mathbf{F})=-\frac{F_{0} D_{0}}{v^{3}}\left[\alpha\left(r_{p}^{2}+r_{a}^{2}-r^{2}\right)-\omega \sqrt{ } \alpha r_{p} r_{a} \cos i\right]
\end{gathered}
$$

Here $\mathbf{i}, \mathbf{j}, \mathbf{k}$ are the unit vectors along the axes, $x, y, z$.

Along with the equations for $r_{p}$ and $r_{a}$ characterizing the dimensions and the compression of the orbit we find the equations for variables $i, \Omega$ and $\varpi$ defining the orientation of the orbit:

$$
\begin{aligned}
i & =\frac{(\mathbf{r} \times \mathbf{F}) \partial \mathbf{c} / \partial i}{m c^{2}}=-\frac{F_{0} D_{0} \omega r^{2} \cos ^{2} u \sin i}{m c v^{3}} \\
\dot{\Omega} & =\frac{(\mathbf{r} \times \mathbf{F}) \partial \mathbf{c} / \partial \Omega}{m c^{2}}=-\frac{F_{0} D_{0} \omega r^{2} \sin u \cos u \sin ^{2} i}{m c v^{3}}
\end{aligned}
$$

For the change of $\varpi$ we have (Subbotin, 1968):

$$
\dot{\varpi}=-(\dot{\varphi})-\cos i \dot{\Omega}
$$

where $\dot{\Omega}$ has been defined by relation (18b), and for the first term in the right-hand side (19) from the equation of the trajectory and the momentum integral we find:

$$
(\dot{\varphi})=\frac{q^{2}}{2 \alpha^{1 / 2} \kappa r_{p} r_{a} r^{2}}\left[\frac{r^{2} \sin 2 \varphi}{q^{4}}\left(q^{2} \dot{c}-2 c q \dot{q}\right)-\sin 2 \varphi \dot{c}+(\mathbf{r} \cdot \mathbf{F}) \frac{\cos 2 \varphi}{m}\right] .
$$

The notation $\dot{\varphi}$ in (19) and (20) indicates that the derivative $\dot{\varphi}$ should be calculated with respect to time entering in the osculating orbital parameters only. For $i$ and $\dot{q}$ according to (13) and (11) we have

$$
\begin{aligned}
& \dot{c}=\alpha^{1 / 2}\left(\dot{r}_{p} r_{a}+r_{p} \dot{r}_{a}\right), \\
& \dot{q}=\left(\frac{2}{r_{p}^{2}+r_{a}^{2}}\right)^{1 / 2} \frac{\left(\dot{r}_{p} r_{a}+r_{p} \dot{r}_{a}\right)-r_{p} r_{a}\left(r_{p} \dot{r}_{p}+r_{a} \dot{r}_{a}\right)}{r_{p}^{2}+r_{a}^{2}} .
\end{aligned}
$$

The relations (16), (18) and (19) obtained above are the differential equations of interest for the osculating orbital parameters.

To estimate the evolution of the orbit under the influence of dynamical friction it is necessary to find the changes of the osculating parameters of the orbit for one revolution of the star around the center of the cluster. Approximate values of these changes can be found by integrating the right-hand sides of the above differential equations with respect to time. So, for instance, the change of $r_{p}$ for one revolution is found with the formula

$$
\Delta r_{p}=r_{p}(\tau)-r_{p}(0)=\int_{0}^{\tau} \mathfrak{f} \mathrm{d} t=c^{-1} \int_{0}^{2 \pi} \mathfrak{f} r^{2} \mathrm{~d} \varphi
$$


where $f$ is the right-hand side of Equation (16) for $r_{p}$. The changes of the remaining elements can be obtained in exactly the same way. While calculating the integrals of the type (21) it is necessary to take into account that the integrands are $\pi$-periodic with respect to $\varphi$ and, moreover, possess the properties of evenness or oddness. The use of these properties enables us to reduce the interval of the integration by a factor 4 and avoid the calculation of the integrals which are equal to zero. In particular, it turns out that

$$
\Delta \varpi=-\cos i \Delta \Omega .
$$

It should be mentioned that the right-hand sides of the equations for $i$ and $\Omega$ keep the angular velocity of the rotation of the cluster $\omega$ as a factor. If $\omega$ is zero, that is, the cluster does not rotate, the parameters $i$ and $\Omega$ are constants, and the motion takes place in an invariable plane. The changes $r_{p}$ and $r_{a}$ in this case do not depend on $i, \Omega$ and $\varpi$. In the general case of the rotating cluster the quantities $i, \Omega$ and $\varpi$ vary with time, and $r_{p}, r_{a}$ depend on $i$ and $\varpi$, but all these quantities do not depend on $\Omega$ (the problem is an axially symmetrical one).

The changes of the orbital parameters for one revolution of the star around the center of the cluster were obtained by numerical estimation of the integrals of type (21) for a number of the values of the parameters $i, \varpi, \omega / \alpha^{1 / 2}$ and for different values of the ratio $m / \bar{m}$ in two cases, namely, that of the globular cluster and that of the open cluster. To be more definite, we assume that the test star moves at the periphery of the cluster. According to the virial theorem we find $\sigma^{2}=0.5 G N \bar{m} \varrho^{-1}$, where $\varrho$ denotes the radius of the cluster. Besides (Chandrasekhar, 1943a), $L=0.55936 D_{0}^{-1 / 3}$. As a typical example let us take the following values of the main parameters for the globular cluster: $N=3 \times 10^{5}, \bar{m}=m_{\odot}, \varrho=35 \mathrm{pc}, r_{p}=27 \mathrm{pc}, r_{a}=30 \mathrm{pc}$. For the open cluster we accepted the values $N=3 \times 10^{2}, \bar{m}=m_{\odot}, \varrho=3.5 \mathrm{pc}, r_{p}=2.7 \mathrm{pc}$ and $r_{a}=3 \mathrm{pc}$.

The corresponding numerical values of $\Delta r_{p}, \Delta r_{a}$ and $\Delta i$ are given in Table I for a number of variants: Due to lack of space the full volume of data obtained is not presented here and we shall restrict ourselves to some qualitative conclusions. The computations show some peculiarities in the evolution of the star orbits. First of all one notices that $r_{p}$ and $r_{a}$ decrease in all cases under consideration and that $r_{a}$ decreases faster than $r_{p}$. This means that the dimensions of the orbit decrease, and the orbit tends to become circular. The changes of $r_{p}$ and $r_{a}$ in the rotating cluster almost always surpass the corresponding values for the nonrotating cluster at least in the case of the direct motion of the test $\operatorname{star}\left(0 \leqslant i \leqslant \frac{1}{2} \pi\right)$. In the rotating cluster the greatest are the changes of $r_{p}$ and $r_{a}$ in the case $i=0$, that is, for the motion in the equatorial plane of the cluster. The changes of $r_{p}$ and $r_{a}$ depend essentially on the magnitude of the ratio $m / \bar{m}$ of the mass of the test star to the average mass of the cluster stars. When the value of this ratio increases the changes of $r_{p}$ and $r_{a}$ increase too.

Another important peculiarity of the evolution of the orbit in the rotating cluster is the decrease of inclination with the passage of time. The velocity of decrease of inclination is greatest for the polar orbits $\left(i=\frac{1}{2} \pi\right)$, while for the equatorial orbits it becomes zero. The magnitude of the change of the inclination is quite significant. As 
TABLE I

Changes of orbital parameters $r_{p}, r_{a}, i$ per one orbital revolution of the test star with $m=4 \bar{m}$ due to dynamical friction in a cluster (abbreviations: $\mathrm{H}$ homogeneous, $\mathrm{E}$ exponential, $\mathrm{O}$ open, $\mathrm{G}$ globular)

\begin{tabular}{llllll}
\hline Type of cluster & $\omega^{\mathrm{a}}$ & $i^{\mathrm{b}}$ & $-\left(\Delta r_{p} / r_{p}\right) \times 10^{4}$ & $-\left(\Delta r_{a} / r_{a}\right) \times 10^{4}$ & $-\Delta i \times 10^{5}(\mathrm{rad})$. \\
\hline HO & 0 & 0 & 534 & 660 & 0 \\
HO & 0.1 & 0 & 633 & 810 & 0 \\
HO & 0.1 & $\frac{1}{2} \pi$ & 531 & 653 & 50.2 \\
& & & & & \\
HG & 0 & 0 & 2.31 & 2.85 & 0 \\
HG & 0.1 & 0 & 2.87 & 3.50 & 0 \\
HG & 0.1 & $\frac{1}{2} \pi$ & 2.30 & 2.82 & 2.17 \\
EO & 0 & 0 & 353 & 575 & 0 \\
EO & 0.1 & 0 & 425 & 677 & 0 \\
EO & 0.1 & $\frac{1}{2} \pi$ & 351 & 573 & 133 \\
& & & & & 0 \\
EG & 0 & 0 & 1.52 & 2.48 & 0 \\
EG & 0.1 & 0 & 1.84 & 2.93 & 0.58 \\
EG & 0.1 & $\frac{1}{2} \pi$ & 1.51 & 2.47 &
\end{tabular}

a The angular velocity of the cluster $\omega$ is taken in terms of the mean orbital motion of the test star.

${ }^{b}$ For the nonequatorial orbits the argument of pericenter $\varpi$ is taken to be zero.

a result of such evolution the plane of the orbit tends step by step to coincide with the equatorial plane of the cluster. The speed of the change of the inclination depends essentially both on the value of the ratio $m / \bar{m}$ and on the initial value of $\varpi$. As to the changes of $\Omega$ and $\varpi$ they depend on the quantity $\varpi$ too and in the order of magnitude they are comparable with the changes of the inclination.

The numerical data of Table I show also that the changes of the orbital parameters in an open cluster are noticeably greater than in a globular one.

All this entitles us to draw the following conclusions: Dynamical friction effects on the star orbits in a rotating homogeneous spherical cluster contribute to concentrating the massive stars both in the vicinity of the center of the cluster and in the equatorial plane of the cluster. The spherical spatial distribution of the stars must transfer step by step to the ellipsoidal one.

\section{The Motion of the Star in a Rotating Spherical Cluster with the Exponential Distribution of the Density}

The observations show that numerous classes of stellar systems, for instance, globular systems, are characterized by a considerable gradient of the stellar density from the center to the periphery. The numerical experiments (Agekjan and Baranov, 1969; Baranov, 1970) also confirm that in the central part of the spherical cluster the density is almost unchanged and then sharply falls towards the periphery. In our previous papers the distribution of the stellar density is traced as far as the boundary of the cluster, where approximately 0.9 of the whole mass of the system is concentrated 
inside the sphere with the radius 0.1 of the radius of the cluster. Therefore, in the motion of stars in such clusters the attraction of the central mass is of crucial significance.

Let us consider a spherical cluster uniformly rotating with a small angular velocity around a fixed axis and assume that the distribution of the stellar matter in the system is subject to the barometrical formula

$$
D=D_{0} \exp \left(-R / R_{0}\right),
$$

where $R$ is the distance of the test star from the center of the cluster, and $D_{0}$ and $R_{0}$ are the constants. The potential $U$ corresponding to the barometrical formula (23) is easily determined from Poisson's equation:

$$
U=-4 \pi G \bar{m} D_{0} R_{0}^{2}\left\{\exp \left(-R / R_{0}\right)+\left(2 R_{0} / R\right)\left[\exp \left(-R / R_{0}\right)-1\right]\right\} .
$$

In particular, it follows from the formula (24) that for large $R$ the field of force becomes similar to the Newtonian one.

Since in the problem under consideration the main attraction force acting on the star moving in the periphery of the cluster is created by its central mass, one can conveniently investigate the motion by classical methods of celestial mechanics using Kepler's osculating elements $a, e, \Omega, i, \varpi, M_{0}$. The differential equations for the osculating Kepler's elements are taken in Euler's form. The right-hand sides of these equations are expressed by means of the components of the disturbing acceleration $S$, $T$ and $W$, which are the projections of the vector of the disturbing acceleration to the directions along the radius-vector, perpendicularly to the radius-vector in the orbital plane and along the normal to the orbital plane, respectively. Since these equations are well-known (see, for example, Subbotin, 1968), they are not listed here.

The star orbits lie in the plane passing through the center of the cluster. The orbits are not closed and cannot be expressed in the elementary functions.

Besides the central mass and the disturbing force of dynamical friction the attraction of the peripheral part of the cluster also influences the star motion. Since we assume the distribution of mass in the system to be spherical, the disturbing force due to the attraction of the peripheral part of the cluster is directed along the radiusvector. The attraction of the peripheral part of the cluster may be described as the difference between the attraction of the mass bounded by the sphere passing through the test star and the attraction of some reference sphere. In the following we assume the undisturbed semimajor axis $a$ to be the radius of the reference sphere. The vector equation of the motion of the test star takes the form

$$
\ddot{\mathbf{R}}=-\frac{G \mathfrak{M}(a)}{R^{3}} \mathbf{R}-\frac{G \Delta \mathfrak{M}}{R^{3}} \mathbf{R}+\frac{\mathbf{F}}{m}
$$

where $\mathfrak{M}(a)$ is the mass of the sphere of the radius $a, \Delta \mathfrak{M}$ is the mass of the spherical layer between the spheres with radii $R$ and $a, \mathbf{F}$ is the vector of the force of dynamical friction defined by formula (7). 
The absolute value of the disturbing acceleration $Q_{R}$ due to the attraction of the peripheral part of the cluster is determined by the formula

$$
Q_{R}=G \Delta \mathfrak{M} / R^{2},
$$

where

$$
\begin{aligned}
\Delta \mathfrak{M}=-4 \pi \bar{m} D_{0} R_{0}\left\{\exp \left(-R / R_{0}\right)\left[\left(R+R_{0}\right)^{2}+R_{0}^{2}\right]-\right. \\
\left.\exp \left(-a / R_{0}\right)\left[\left(a+R_{0}\right)^{2}+R_{0}^{2}\right]\right\}
\end{aligned}
$$

The components $S, T$ and $W$ of the disturbing acceleration due to the force of dynamical friction take the form

$$
\begin{aligned}
& S=-\frac{F_{0}}{m} \frac{D}{v^{3}} \sqrt{\left(\frac{\mu}{p}\right) e \sin f,} \\
& T=-\frac{F_{0}}{m} \frac{D}{v^{3}}\left[\sqrt{ }\left(\frac{\mu}{p}\right)(1+e \cos f)-\omega R \cos i\right] \text {, } \\
& W=-\frac{F_{0}}{m} \frac{D}{v^{3}} \omega R \sin i \cos (f+\varpi),
\end{aligned}
$$

where

$$
\begin{aligned}
v=\left\{\frac{\mu}{p}\left(1+2 e \cos f+e^{2}\right)\right. & -2 \sqrt{ }\left(\frac{\mu}{p}\right)(1+e \cos f) \omega R \cos i+ \\
& \left.+\omega^{2} R^{2}\left[\cos ^{2} i+\sin ^{2} i \cos ^{2}(f+\varpi)\right]\right\}^{1 / 2},
\end{aligned}
$$

$p=a\left(1-e^{2}\right), f$ is the true anomaly, $\mu$ is the product of the constant of gravitation on the mass of the central part of the cluster. When deriving (27), the formulae by Fominov (1963) were used. In these formulae the absolute value of the disturbing resistance force was taken in the form (7).

Introducing now the eccentric anomaly $E$ as the independent variable into the differential equations of the motion according to the formula

$$
\frac{\mathrm{d} t}{\mathrm{~d} E}=\frac{a^{3 / 2}}{\mu^{1 / 2}}(1-e \cos E),
$$

taking into account the well-known relations

$$
\begin{aligned}
& R \cos f=a(\cos E-e), \\
& R \sin f=a \sqrt{1-e^{2}} \sin E,
\end{aligned}
$$

and the relations (25), (25)-(28) and integrating numerically the right-hand sides of the equations of motion for one revolution of the star around the center of the cluster, 
we obtain the estimates of the changes of the elements for one revolution. We accept $a=30 \mathrm{pc}, e=0.2$ for the globular cluster and $a=3 \mathrm{pc}, e=0.2$ for the open cluster. The angular velocity of the rotation is taken to $b e \omega=0.1 n$, where $n$ is the mean motion of the test star. Some numerical data are given in Table I.

The results demonstrate the complicated character of dynamical friction effects on the evolution of the orbit. One notices that the dimensions of the orbit decrease, and the orbit tends to become circular. The changes of $r_{p}$ and $r_{a}$ in the rotating cluster surpass the corresponding changes of these elements in the nonrotating cluster. In the rotating cluster the greatest deformation is of the orbit of the test star moving in the equatorial plane $(i=0)$.

The changes of the orbital elements increase noticeably when the ratio $m / \bar{m}$ increases. The changes of the elements in an open cluster are considerably greater than in a globular cluster.

An important peculiarity of the evolution of the orbit in the rotating cluster is the decrease of orientation with passage of time. Since the right-hand sides of the equations of motion for $i$ and $\Omega$ contain the angular velocity of the rotation of the cluster $\omega$ as a factor, in the nonrotating cluster $(\omega=0)$ the changes of $i$ and $\Omega$ are equal to zero, that is, the test star moves in an invariable plane. The changes of the dimensions in this case are not dependent on $i, \Omega$ and $\varpi$. In the general case of the rotating cluster $i$, $\Omega$ and $\varpi$ vary with time, and $r_{p}$ and $r_{a}$ depend on $i, \varpi$ (the dependence of $r_{p}$ and $r_{a}$ on $\varpi$ is quite weak), but all these parameters do not depend on $\Omega$.

In the case under consideration (in quite the same way as in the case of the homogeneous cluster) the angles $\Omega$ and $\tilde{\omega}$ are connected by Equation (22). This follows from the properties of evenness (oddness) and periodicity of the right-hand sides of the equations of motion.

The change of inclination is greater than the changes of other elements defining the orientation of the orbit. The change of $i$ is maximum for the polar orbits $\left(i=\frac{1}{2} \pi\right)$, while for the equatorial orbits $(i=0)$ it becomes zero. The changes of $\Omega$ and $\varpi$ are comparable in the order of magnitude with the changes of inclination. The changes of $i, \Omega$ and $\varpi$ depend essentially on the initial value of the parameter $\varpi$.

Thus the evolution of the orbits in the rotating cluster with the exponential distribution of the density tends to concentrate the massive stars both near the equatorial plane of the cluster and near the center of the cluster.

\section{Conclusions}

The estimation of dynamical friction effects on the orbit of the test star under the influence of the regular gravitational field and dynamical friction shows that in stellar systems with relatively short times of relaxation dynamical friction effects are not negligible. Both density distributions in the cluster are considered: the homogeneous and the exponential ones. The numerical examples. show that the orbit of the test star in the cluster, in general, is subject to complicated variations: the originally elliptic orbit tends to become circular and its axis and inclination decrease with pas- 
sage of time. Due to the influence of dynamical friction the massive stars tend to concentrate both in the vicinity of the center of the cluster and of the equatorial plane of the cluster.

\section{References}

Agekjan, T. A. and Baranov, A. S.: 1969, Astrofiz. 5, 305.

Baranov, A. S.: 1970, Astrofiz. 6, 261.

Chandrasekhar, S.: 1942, Principles of Stellar Dynamics, The University of Chicago Press, Chicago.

Chandrasekhar, S.: 1943a, Rev. Mod. Phys. 15, 1.

Chandrasekhar, S. : 1943b, Astrophys. J. 97, 255 (Parts I, II); 98, 54 (Part III).

Fominov, A. M.: 1963, Bull. Inst. Theor. Astron. 9, 185 (in Russian).

Rosenbluth, M. N., MacDonald, W. M., and Judd, D. L.: 1957, Phys. Rev. 107, 1.

Subbotin, M. F.: 1968, Vvedenie v Teoreticheskuyu Astronomiyu, Moscow.

\section{DISCUSSION}

R. H. Miller: When inclined orbits tend to decrease their inclinations, approaching the equatorial plane, do they approach monotonically, or can they overshoot and approach in a damped oscillation mode?

A. S. Baranov: They approach monotonically. 